\title{
AKKADISCHES LEHNWORTGUT IM HURRITISCHEN
}

Das Hurritische enthält bekanntlich eine größere Anzahl von Lehnwörtern aus dem Akkadischen, der damaligen internationalen Verkehrs- und Diplomaten- sprache. Darauf hat in größerem Umfang schon E. Laroche aufmerksam gemacht'. Auch die in KBo 32 edierte $^{2}$, in StBoT 32 bearbeitete hurritisch-mittelhethitische Bilingue $^{3}$ bietet eine stattliche Reihe von akkadischen Lehwörtern, die, nach Bedeutungsgruppen gegliedert, nun Gegenstand dieser kleinen Abhandlung sein sollen.

Aus folgenden Bedeutungsgruppen lassen sich innerhalb der Bilingue hurritische Wörter akkadischer Provenienz ausmachen:

1. Hurritische Wörter, die nach Auffassung von E. Laroche dem Akkadischen entlehnt (vgl. sarri "König") oder von einem akkadischen Wurzelelement abgeleitet sind (vgl. hurr. puhugari "Tausch, Ersatz, Substitut"), lassen sich leicht dem Index "Akkadien" seines Glossaire de la langue hourrite (1980) entnehmen, in welchem die akkadisch-hurritischen Wortent sprechungen übersichtlich aufgelistet sind (315-316). Vgl. Verf., BAC 23, 1995, 36 Anm. 39.

2. Die in KBo 32 (Berlin 1990) enthaltenen Autographien dieser Bilingue wurden von $\mathrm{H}$. Otten und $\mathrm{Chr}$. Rüster angefertigt. Bei Zitaten aus der Bilingue werden in diesem Beitrag die Textnummern ohne Angabe des Editionsbandes angefuihrt.

3. E. Neu, Das hurritische Epos der Freilassung I- Untersuchungen zu einem hurritisch-hethitischen Textensemble aus Hattuša (=StBoT 32), 1996. Für den zweiten Band (StBoT 33) sind eine auf dem hurritischen Text der Bilingue erstellte hurritische Grammatik sowie ein ausführliches Glossar mit den hurritisch-hethitischen Wortentsprechungen vorgesehen. Für den hurritischen Wortschatz der Bilingue sei auch verwiesen auf J. Catsanicos, L'apport de la bilingue de Hattuša à la lexicologie hourrite, Amurru 1, 1996, 197-296, der für hurritische Wortformen, die ihm aus dem Akkadischen entlehnt zu sein scheinen, jeweils auch die akkadische Entsprechinf anführt. 


\section{BAUWESEN}

alibši (a-li-ib-ši) 14 Rs. 40 "Lehmziegel”" (hethit. sumerograph. $\mathrm{SIG}_{4}$ ). Das hurritische Wort könnte in Anlehnung an akkad. $l i b=i t t u$ "Ziegel, Ziegelwerk" gebildet sein, mit nominalem Ableitungssuffix $-s i$ des Hurritishen und prothetischem $a$ - (vgl. hurrit. izüzi gegenüber akkad. zizu "Emmer"); StBoT 32, $195 f$.

haikalli ([h]a-i-kal-li, ha-a-i-kal-li) 13 I 2; 212 Ik. Kol. 3' "Palast" (heth. Ehalentuua) könnte vielleicht über eine semitische Sprache letztlich auf sumer. é . g a I zurückgehen (StBoT 32, 228 ff. $)^{4}$.

hirìdi (hi-ri-i-ti) 14 Rs. 35 "Baugrube" aus akkad. hirītu "Graben" (StBoT 32, 185f.).

idenni (i-te-en-ni) 14 Rs. 38 "Baumeister". Die Herkunft aus akkad. itinnu/etennu ist unverkennbar (StBoT 32, 183; FsNeve 1994, 59ff.).

mahiri (ma-a-hi-ir-ri, mit 'Artikel') 19 I 32; 214 I 5' (ohne Pleneschreibung in der ersten Silbe) "Marktplatz" hat man zu akkad. mahiru zu stellen (StBoT 32, 426; E. Laroche, GLH 165).

$\check{s} \bar{u} g i(\tilde{s} u-u ́-k i) 14$ Rs. 40 bezeichnet die Dachkonstruktion eines Turmes und könnte vielleicht mit akkad. šŭqu "Höhe" zu verbinden sein (StBoT 32, 195). Ausdrücke, die ein - bestimmtes

4. W.E.G. Watson (Brief vom 2.4. 1997) äuBert hinsichtlich dieser Herleitung Bedenken gegenüber einer Gleichsetzung von hurr. $\underline{h}$ mit semit. $\underline{h}$

5. Abweichende Lesung des hurritischen Wortes bei V. Haas-l. Wegner, FsNeve 1994, 54, 56.- Unsere interpretierende Lesung als hiridi, also mit -d-statt akad. -\% folgt der Sturtevantschen Regel. Entsprechendes gilt für idenni, šügi u.a.m. (s. im folgenden).- In StBoT 32, 186 muB es in der zweiten Zeile hutanu-, in der dritten Zeile ḩusanuš, in der fünften Zeile von unten als Übersetzung von havurunni aber "der Himmel" heißen.

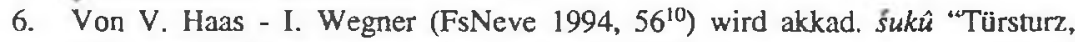
Türstange" (W. von Soden, AHw III 1265) herangezogen, was jedoch semantisch schwierig ist. Von der lexikalischen Entsprechung des Hethitischen ist nur -ar erhalten, das darauf folgende Element $-s i$ stellt entgegen den beiden Autoren den Dativ des Personalpronomens der 3. Pers. Sing. dar ("ihm, ihr"). 
Befestigungswerk einer Stadt betreffen, scheinen hingegen dem Hurritischen zu entstammen und wurden akkadisiert, wovon hurr. adašši (a-ta-aš-ši-i, a-ta-aš-še) 19 I 36-39 "Umwallung der Unterstadt" und kirhhi (ki-ir-hi) 19 I 36 "Umwallung der Oberstadt" beredtes Zeugnis ablegen. Das Wort adašši zeigt im Ausgang das weit verbreitete hurritische Nominalsuffix -ši (s. auch schon oben alibši $i$, das abstrakte und kollektivische Funktion haben kann. Die

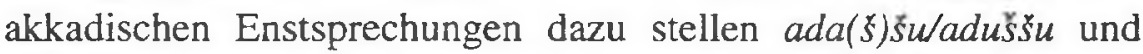
kirhu/kerhu dar (StBoT 32, 425) dar und zeigen hinsichtlich ihres Auftretens eine dafür typische regionale Verbreitung (V. Haas-I. Wegner, FsBoehmer 1995, 191ff.) ${ }^{7}$. Der hethitische Übersetzer gibt die hurritischen Ausdrücke adassi und kirhi behelfsweise mit URU-aš katteraš uahnueššar "Unwallung der Unterstadt" und šarazzịaš uahnnueššar "Umwallung der Oberstadt" wieder (StBoT $32,432)$.

\section{VERWALTUNG}

halzi (hal-zi, hal-zé, hal-zé-e) 14 I 34, 37, 33 "Disktrikt, Verwaltungsbezirk" gehört ohne Zweifel zu akkad. halsu "Festung", doch scheint im Hurritischen diese Bedeutung in den Hintergrund zu treten; für hurrit. halzi (halsi) ließe sich allenfalls noch die Bedeutung "Grenzbezirk" erwägen (StBoT 32, 134f.).

halzuhli (hal-zu-u-uh-la Essiv) 14 I 32, 33 stellt das Nomen agentis zum vorhergehenden Ausdruck halzi dar und läßt sich als "Distriktverwalter, Distriktaufseher" deuten. Akadisiert erscheint dieses Wort als hals/zuhlu (W. von Soden, AHw I ${ }^{2}$ 314a). Der hethitische Übersetzer gibt hurrit. halzuhli mit auriaš išha- (wörtlich:) "Herr der Warte" wieder, was zunächst militärisch zu verstehen ist ("Grenzkommandant" o.ä.), dann aber, wie in der Bilingue, mehr im verwaltungstechnischen Sinne den "Distriktaufseher" meint.

7. Mit V. Haas-I. Wegner (a.a.O. 191) halte ich die Annahme, wonach adaššsi eine Ableitung von ardi "Stadt" darstellen soll (G. Wilhelm, Orientalia 61, 1992, 128, 135) für unwahrscheinlich. $\mathrm{Zu}$ ada( $(\tilde{s}) \operatorname{su}(m)$ und $k i r h u(m)$ s. auch $\mathrm{J}$. Catsanicos, Amumu 1, 1996, 238.

8. G. Wilhelm (Orientalia 61, 1992, 130) schreibt "(Militär-) Bezirk". 
haziijani (ha-zi-ia-na Essiv) 14 Rs. 25, aus akkad. haziänu entlehnt, läßt sich daher als "Bürgermeister" deuten, wobei das lexikalische Pendant im Hethitischen ( ${ }^{U}$ utniiašha-) wörtlich als "Landesherr" zu übersetzen ist (StBoT 32, 182) .

šarri (šar-ri) 133 I 3 "König”, akkad. šarru". Seine Entsprechung in der hethitischen Übersetzung ist LUGAL- $u \grave{s}$, dieses Sumerogramm kann aber auch das genuin hurritische Wort wiedergeben: evrinna = LUGAL MEŠ 19 I 6, 8, II 6, 7, wobei evri allerdings zunächst nur "Herr" bedeutet, doch s. auch talavuši evri = LUGAL GAL "großer König" (15 IV/UI 14).

*teli (in: tel-li-(i-)ip-pa) 14 Rs. 15, 25, dem in der hethitischen Übersetzung das Sumerogramm IGI.DU ${ }_{8}{ }^{\text {HI.A }}$ "Abgaben" entspricht, läßt sich mit akkad. $t e \bar{l} \bar{l}=t u$ "Ertrags(abgabe)" zusammenstellen (StBoT 32, 173f.).

\section{MAßBEZEICHNUNGEN}

parissade (pa-ri-is-sà-te) 15 I 9', 11" “1/2 Kor" enthält akkad. paris $(=u)$ und das auch bei anderen Maßbezeichnungen " wie auch bei Zahlen $^{12}$ aftretende kollektivische Suffix -ad- mit auslautendem Stammvokal (StBoT 32, 309; Das Hurritische, 16 ${ }^{42}$ ).

šiklade (ši-ik-la-te) 15 I 6', 7' "1 Schekel" beruht auf akkad. śiql(u) und ist um das Ableitungssuffix -ad-erweitert (StBoT 32, 305).

Umgekehrt kann auch ein genuin hurritisches Flächenmaß akkadisiert auftreten, wie aviharu zeight (W. von Soden, AHw I', 90a), das innerhalb der Bilingue im Genitiv mit 'Artikel' bezeugt ist (13 I 5; StBoT 32, 242).

9. Vgl. F. Starke, ZAR 2, 1996, 159.

10. Der Begriff "König" wird hier der Bedeutungsgruppe VERWALTUNG im weitesten Sinne zugeordnet.- Neben der Schreibung šar-ri gibt es auch die Graphie ša-ar-ri: 20 I 6', vgl. 3', 18'; 209, 12'.

11. Vgl. šiklade (s. im folgenden), šahatnadi 15 I 7" "ein halber Schekel" (so muB es auch in StBoT 32, 289 Vs. II 7' richtig heißen) oder suuatatte 15 I 10' "ein Viertel Kor" mit ungewöhnlicher Doppelschreibung des Dentals.

12. Vgl. tu-um-na-ti (tumnadi) vier bzw. "Gruppe von Vier" (13 I 30). 


\section{GETREIDEBEZEICHNUNGEN}

$i z \bar{u} z i$ ( $i-z u-u$-zi) 15 I 10' "Emmer" ist aus akkad. zizzu umgebildet (Metathese mit prothetischem $i-$ ); vgl. StBoT 32, 310 f.

ubi (ú-bi) 15 I 11' "Gerste" klingt an die akkadische Saatmaßbezeichnung ubû an (StBoT 32, 309).

utte (ut-te) ist innerhalb der Bilingue nicht bezeugt, hier nur der Vollständigkeit halber hinzugefügt, geht auf akkad. uttetu "Gerste, Getreide; Korn" zurück (StBoT 32, 311).

Eine genuin hurritische Getreidebezeichnung liegt in kade vor (StBoT 32, 309).

\section{BROTBEZEICHNUNG}

kakkari $\left(k a_{4}-a k-k a_{4}-r i\right) 14$ Rs. 23 dürfte aus dem Semitischen entlehnt sein (vgl. akkad. kakkarw/kakkartu "Rundbrot") und wird in der hethitischen Übersetzung mit ${ }^{N I N D A}$ kugulla- wiedergegeben, das allerdings dort auch hurrit. šullübri entspricht (StBoT 32, 166f. 179f.) ${ }^{13}$.

\section{TIERBEZEICHNUNGEN}

kunkalle (ku-un-kal-le-e) 13 I 17 "Fettschwanzschaf"; vgl. akkad. g/kukkallu (StBoT 32, 255).

nāli (na-a-li) 14 I 1, 12, 17 "Reh(bock)" hat man zu akkad. nälu/na (j)jalu zu stellen (StBoT 32, 99).

serre (se 20 -er-re-e) 14 Rs. 55 "Esel" (hethit. sumerograph. ANŠE) wird man zu der in Nuzi belegten Eselbezeichnung z/serru zu stellen haben und etymologisch dafür auch akkad. serrēmu heranziehen dürfen (StBoT 32, 199; J. Catsanicos, Amurru 1, 1996, 244) ${ }^{14}$.

13. Vgl. J. Puhvel, HED 4, 1997, 15f. sub kak(k)ari-, 233f. sub kuk(k)ul(l)a-.

14. Akkad. serrēmu hat ais Wortzeichen ANŚE.EDIN.NA (W. von Soden, AHw II 1038a), so daß auch hurrit. serre nicht den Esel schlechthin (akkad. imêru), sondern einen bestimmten Onagertyp gemeint haben könnte, Für den hethitischen Übersetzer der hurritischen Vorlage spielte diese Differenzierung jedoch keine Rolle, und man begnügte sich mit dem Wortzeichen ANŚE. 
Fraglich bleibt, ob hinter hurrit. kammi eine Vogelbezeichnung steckt und, falls ja, diese dann auf akkad. gamgammu beruht, wie von J. Catsanicos (a.a. O. $207^{51}$ ) vorgeschlagen (vgl. StBoT 32, 180).

\section{HOLZVERABEITUNG ${ }^{15}$}

abri (a-ab-ri) 14 Rs. 56 "Holzstoß"; akkad. abru (StBoT 32, 201).

*šašāri (ša-šar-ri, mit 'Artikel') 14 Rs. 55 "Säge"; akkad. šaššāaru (StBoT 32, 199).

\section{HAUSHALTSBEREICH}

humni (hu-u-um-ni) 19 I 36 "Herd-/Feuerstelle" wird man hinsichtlich seines Wurzelements zu akkad. hum $=\imath u$ "Fieber, Hitze" bzw. hamätu "brennen, verbrennen" stellen dürfen (StBoT 32, 429f., 432). Davon ist hurrit. ephe "Ofen" (hethit. sumerograph. UDUN) inhaltlich zu trennen (StBoT 32, 167). Hier in der Erzählung um die Stadt Ebla hat humni stark symbolhaften Charakter: die Zerstörung der Feuerstelle bedeutet zugleich die Zerstörung der Stadt.

kāsi (ka-a-sí) 12 IV 18'; 14 I 42 "Becher”, akkad. kāsu (StBoT 32, $71,143)^{16}$.

malladi (ma-al-la-ta-el ${ }^{17}$ ) 13 I 23, von uns unter Vorbehalt mit "Gedeck" übersetzt, umfaßt gemäß der hethitischen Übersetzung eine

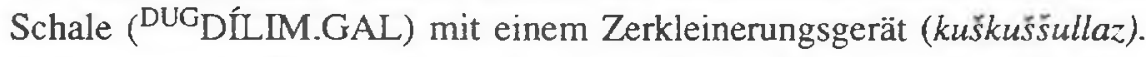
V. Haas erwägt Herkunft aus akkad. mall(atu), jedoch mit hurritischem Wortausgang (StBoT 32, 261) ${ }^{18}$.

15. Im allgemeinsten Sinne. Die hurritische Bezeichnung für "Holz" ist tâli (E. Laroche, GLH 253; Verf., StBoT 32, 198).

16. Ob ein etymologischer Zusammenhang zwischen hurr, hüruffe "Rhyton, TiergefäB" (StBoT 32, 270) und akkad. luuruppu (W. von Soden, AHw I ${ }^{2} 360 \mathrm{~b}$ ) besteht, bleibt fraglich; vgl. J. Catsanicos, Amurru 1, 1996, 243.

17. Instrumental malladae mit enklitischem Pronomen -l(la).

18. Akkad. GiŚ $a t m u \hat{u}$ (Nuzi), das als Fremdwort gilt und dessen genaue Bedeutung bisher unklar war (vgl. CAD AЛI, 1968, 489b), erklärt sich inzwischen aus hurrit. admi "Schemel, Fußbank" (StBoT 32, 242). 


\section{KRIEGSWESEN}

asiri (a-sí-i-ri, a-as-sí-i-ri) 21 I 3'; 19 I 3 "Gefangener" (hethit. appa piianz); akkad. asiru (StBoT 32, 400) ${ }^{19}$.

sallt $^{20}$ "Raub, Beute, Unterschlagung" wird von J. Catsanicos (Amurru 1, 1996, 248) im Anscluß an einen diesbezüglichen Vorschlag von J.-M. Durand zu akkad. šall $=a t u(m)$ gestellt (StBoT 32, 177, 182f.).

til-(ti-lu-lu-u-uš-tab, Fut. Sg. 1.) 19 I 28 "zertrampeln, zerstampfen" könnte lautlich und semantisch mit akkad. $t i l=u(m)$ "Schutt-/Ruinenhügel" in Verbindung zu bringen sein (StBoT 32, 425) ${ }^{21}$.

ulmišši (u-ul-mi-iš-ši) 19 I 16 "Waffen”. Der Ausdruk ulmi=šši stellt eine Kollektivbildung des Wortes ulmi "Waffe" dar, das I. Wegner (AOAT 36, 1981, 99) mit akkad. ulmu verbunden hat (StBoT 32, 441 mit Anm. 37).

Für eine Bewertung der aufgezeigten Bedeutungsgruppen aus akkadischem Lehnwortgut mit Blick auf Wortschatz und Kultur der

19. Aus der Verwendung von hethit. appa piianz (wörtlich:) "der (jemandem) ausgeliefert (ist)" statt Lú appanz "Kriegsgefangener" darf man wohl schließen, $\mathrm{daB}$ mit hurrit. asīi nicht ein Kriegsgefangener gemeint ist. Für akkad. asïru finden sich im CAD A/II, 1968, 331a die Bedeutungen "prisoner of war" und "captive foreigner used as worker", W. von Soden (AHw I ${ }^{2}$ 74a) führt als Bedeutung "(Kriegs-) Gefangener" an. Die zweite Bedeutungsangabe des CAD ("captive foreigner used as worker") würde für die Gefangenen bzw. Unfreien innerhalb der bilingue recht gut passen, da diese Sklavendienste im Haus (Kochen, Auftragen von Essen und Trinken, Abwaschen) wie auch bei der Textilienherstellung zu erfüllen haben (15 I 26' -IV 2, StBoT 32, 292/293, 294/295). Bei der Versammlung der zehn Könige haben Sie für deren Verköstigung zu sorgen (19 I 3-8; StBoT 32, 378/379).

20. Hurrit. $\breve{s} a l l i$ ist innerhalb der Bilingue nur im Instrumental bezeugt: sal-la-e(-na), ša-a-al-la-e(-na) 14 Rs. IV 18, R. 27. Dieses Wort tritt dort im Verwaltungsbereich auf.

21. Möglicherweise liegt auch in einem anderen Falle Anleihe bei einem akkadischen Verbum vor. So vermutet J. Catsanicos (Amurru 1, 1996, 275220) eine Verbindung zwischen dem intransitiven hurritischen Partizip ši-pa-a $/ Y_{i b}=a /$ "ausgetrocknet, ausgedörtt" (15 I 14') und akkad. šabābu(m) "glühen, verdorren" bzw. šibūbu "Funke (?)"; zu hurrit. ši-pa-a vgl. StBoT 32, 319. 
Hurriter ist es gewiß noch zu früh, zumal da wir uns textlich auf die in Nordsyrien geschaffene hurritische Version der Bilingue beschränkt haben. Bemerkenswert sind aber schon jetzt die Bereiche Bauwesen und Verwaltung unter Einschluß der Maß- und Getreidebezeichnungen. Daß die Hurriter nach ihrer Einwanderung von Nordosten in ihre späteren Siedlungsgebiete des Vorderen Orients und mit ihrer Integration in die dort herrschende Keilschriftkultur Anleihe am Wortschatz des damals weit verbreiteten Akkadischen machten und zusammen mit den Sachen auch die entsprechenden Wörter übernahmen, ist nicht ungewöhnlich ${ }^{22}$. Zwei genuin hurritische Termini des Fortifikationswesens zeigen, daß die Hurriter nicht nur die Nehmenden waren, wie sich dies außerhalb der Bilingue in hurritisch dominierten Regionen (z.B. Nuzi) häufiger beobachten läßt. Mit ihren zahlreichen Mythen und Erzählstoffen, von denen einige ihren Wege auch zu dem frühen Griechentum fanden, haben sie ohnehin die geistige und religiöse Welt des Alten Orients wesentlich bereichert, wobei die diesem Beitrag zugrunde gelegte hurritische Fassung der zweisprachigen Textgruppe aus KBo 32 mit ihren menschlichen Verhaltensmustern, die Ausfluß einer umfangreicheren Weisheitsliteratur sein dürften, ohne Zweifel einen gewissen literarischen Höhepunkt bildet.

\section{LITERATUR}

Catsanicos, Jean, L'apport de la bilingue de Hattusa à la lexicologie hourrite, in: Amurn 1, 1996, 197-296.

[Chicago Assyrian Dictionary]. The Assyrian Dictionary of the Oriental Institute of the University of Chicago [CAD]. Chicago-Glückstadt 1956ff.

22. Es ist $z u$ erwanten, daB sich im Laufe der Zeit noch für manch anderes hurritische Wort ein akkadisches Etymon wird erweisen lassen. So klingen etwa hurrit. nav- "weiden" und navni "Weide" an akkad. nawûm "Weidegebiet, Steppe" (W. von Sohen, AHw II 77la; Verf., StBoT 32, 105) an. Das hurritische Demonstrativpronomen [a-ag-]ga-an-ni (StBoT 32, 134) ähnelt dem akkadischen Demonstrativum agannî, doch benht letzteres auf einer Zusammensetzung (AHw I ${ }^{2}$ 15b). Hurrit. * "serhi "behaartes Fell, (eventuell auch) Mähne", von dem in der Bilingue nur der quativ śerbuš bezeugt ist, wird man vielleicht unter Annahme einer Metathese mit akkad. še'ru "haarig" (CAD Š/II, 1992, 327) verbinden dürfen (vgl. StBoT 32, 343). Zu dem dort von uns erwähnten Wort aus Nuzi s. aber auch CAD a.a.O. 315a sub šerhatu. 
Haas, Volkert-Wegner, Ilse, Baugrube und Fundament, in: Festschrift fir Peter Neve [FsNeve]. (Istanbuler Mitteilungen 43). Tübingen 1994, 53-58.

Haas, Volkert-Wegner, Ilse, Stadtverfluchungen in den Texten aus Boğazköy sowie die hurritischen Termini fur "Oberstadt", "Unterstadt" und "Herd", in: Beiträge zur Kulturgeschichte Vorderasiens. Festschrift für Rainer Michael Boehmer [FsBoehmer]. Herausgegeben von U. Finkbeiner, R. Dittmann und H. Hauptmann. Mainz 1995, 187-194.

Laroche, Emmanuel, Glossaire de la langue hourrite [GLH]. Paris 1980.

Neu, Erich, Das Hurritische: Eine altorientalische Sprache in neuem Licht, in: Abhandlungen der Geistes- und sozialwissenschaftlichen Klasse (Akademie der Wissenschaften und der Literatur, Mainz). Jahrgang 1988, Nr. 3 Mainz-Stuttgart.

Neu, Erich, "Baumeister" und "Zimmermann" in der Textïberlieferung aus Hattuša, in: Festschrift für Peter Neve [FsNeve]. Istanbuler Mitteilungen, 43). Tübingen 1994, 59-62.

Neu, Erich, Mehrsprachigkeit im Alten Orient-Bilinguale Texte als besondere Form sprachlicher Kommunikation, in: Bochumer Altertumswissenschaftliches Colloquium [BAC], 23. Trier 1995, 11-39.

Neu, Erich, Das hurritische Epos der Freilassung I- Untersuchungen zu einem hurritisch-hethitischen Textensemble aus Hattusa (=Studien zu den Boğazköy-Texten [StBoT], 32). Wiesbaden 1996.

Otten, Heinrich-Rüster, Christel, Die hurritisch-hethitische Bilingue und weitere Texte aus der Oberstadt (= Keilschrifttexte aus Boghazköi [KBo], 32). Berlin 1990.

Puhvel, Jaan, Hittite Etymological Dictionary [HED]. Volume 4: Words beginning with K. Berlin-New York 1997.

Soden, Wolfram von, Akkadisches Handwörterbuch [AHw]. Bd. I ${ }^{2}$ 1985, II 1972, III 1981. Wiesbaden.

Starke, Frank, Zur "Regierung" des hethitischen Staates, in: Zeitschrift für Altorientalische und Biblische Rechtsgeschichte [ZAR] 2, 1996, 140-182.

Wegner, Ilse, Gestalt und Kult der lśtar-Šawuska in Kleinasien (Hurritologische Studien III), in: Alter Orient und Altes Testament [AOAT], 36. Neukirchen-Vluyn, 1981.

Wilhelm, Gernot, Hurritische Lexikographie und Grammatik: Die hurritisch-hethitische Bilingue aus Bogazköy, in: Orientalia (Series Nova) 61, 1992, 122-141. 